\title{
Evaluando un lexicón para la clasificación de polaridad a nivel de Tweet en español
}

\author{
Fabián Paniagua-Reyes, José A. Reyes-Ortiz, Belem Priego-Sánchez \\ Universidad Autónoma Metropolitana, Azcapotzalco, \\ México \\ $\{a 12112002241$, jaro, abps $\}$ @azc.uam.mx
}

\begin{abstract}
Resumen. El análisis de sentimientos es una tarea que representa un reto y que cada día obtiene mayor relevancia en el procesamiento automático de textos. Sin embargo, existe una necesidad latente de contar con recursos de análisis de textos en español como la tarea de clasificación de polaridad. En este artículo se presenta una arquitectura para la evaluación de un lexicón denominado MLSentiCon para la tarea de clasificación de polaridad en tweets en español, utilizando Máquinas de Soporte Vectorial, el modelo de bolsa de palabras y una ponderación de sus entradas mediante la métrica TF-IDF. La evaluación del lexicón se lleva a cabo en tres conjuntos de datos para diferentes cantidades de categorías. Los resultados de la evaluación muestran una perspectiva de comparación para los tres conjuntos.
\end{abstract}

Palabras clave: Lexicón, clasificación de polaridad, análisis de sentimientos, procesamiento de textos en español.

\section{Evaluating a Lexicon for Polarity Classification at Tweet Level in Spanish}

\begin{abstract}
The sentiment analysis is a task that represent a major challenge and, becomes increasingly relevant in text processing. However, there is a latent need to have resources for analyzing texts in Spanish such as polarity classification task. This paper presents an architecture to evaluate a lexicon called MLSentiCon for polarity classification in Spanish tweets, using Support Vector Machine algorithm, the bag-of-words model and a weighting based on TF-IDF metric. The lexicon evaluation is carried out in three data sets for different number of categories. The evaluation results show a comparison perspective for such three sets.
\end{abstract}

Keywords. Lexicon, polarity classification, sentiment analysis, Spanish text processing. 


\section{Introducción}

El análisis de sentimientos es una tarea que se encarga de crear modelos computacionales para procesar textos en lenguaje natural y determinar la carga emocional impresa en el texto. Este análisis abarca diversas tareas como: determinar la polaridad de un texto (positiva, negativa y neutra), identificar la emoción expresada en el texto (alegría, enojo, furia, tristeza, depresión) y establecer el grado de polaridad (muy positivo, positivo, negativo, muy negativo, neutro o nada). Estas actividades se abordan como una tarea de clasificación automática de texto.

El análisis de sentimientos es una tarea que se ha abordado, ampliamente, a partir de textos en inglés. Sin embargo, para el español las propuestas han sido escasas, esto ha ocasionado una carencia de recursos de análisis de opiniones para este idioma. En este aspecto, existen competencias internacionales para evaluar sistemas computacionales para el determinar la polaridad de un mensaje de red social.

Tal es el caso del Taller sobre Análisis Semántico en SEPLN (TASS) en sus diversas ediciones. En la edición del TASS 2017 [1] se presenta una tarea para el análisis de sentimientos a nivel de tweet y el análisis de sentimientos basados en aspectos. La tarea de análisis de sentimientos a nivel de tweet considera cuatro categorías: Positivo (P), Negativo (N), Neutro (NEU) y Ninguna (NONE). Mientras que en la edición del TASS 2016 [2], dicha tarea está catalogada en dos formas, por una parte, seis casos de etiquetas: Muy Positivo (P+), Positivo (P), Negativo (N), Muy Negativo (N+) Neutro (NEU) y Ninguna (NONE). Por otro lado, también existen los casos con cuatro etiquetas: Positivo (P), Negativo (N), Neutro (NEU) y Ninguna (NONE).

El uso de lexicones para la tarea de clasificación de la polaridad de un tweet es, cada día, más común. Los lexicones ofrecen una lista de palabras o lemas polarizadas con las cuales se puede determinar la polaridad de todo un mensaje o texto.

Po lo anteriormente descrito, en este artículo se presenta una arquitectura para evaluar el uso del lexicón denominado $M L$-SentiCon [3] en la tarea de clasificación de polaridad, utilizando los datos de la competencia TASS en sus ediciones 2016 y 2017 en la tarea de análisis de sentimientos a nivel de tweets en español. La tarea de clasificación se lleva a cabo con el algoritmo de Máquinas de Soporte Vectorial y como características, el pesado TF-IDF de los lemas en español del lexicón $M L$-SentiCon.

El resto del artículo se organiza como sigue: en la sección 2, se presenta el estado del arte de trabajos que han realizado el análisis de sentimientos en español con diversos enfoques. La sección 3, expone la arquitectura de evaluación del lexicón para la tarea de clasificación de polaridad. La sección 4, presenta la configuración experimental. Por su parte, la sección 5 expone los resultados de la experimentación realizada. La sección 6 presenta las conclusiones y el trabajo a futuro.

\section{Estado del arte}

El análisis de sentimientos para el español ha sido abordado desde diversos enfoques. Como en [4], [5] y [6], que han técnicas de aprendizaje profundo para representar los tweets y desempeñar el análisis de sentimientos a nivel de sentencias. El uso de vectores 
de características es ampliamente utilizado en la tarea de análisis de sentimientos. En [7] usan vectores de características de baja dimensión para representación del texto, ellos proponen un modelo simple fundamentado en la normalización de texto con identificación de marcadores de énfasis, el uso de modelos de lenguaje para representar las características locales y globales del texto, y características como emoticones y partículas de negación; en [8], del mismo modo, construyen vectores de palabras a partir de la información de opinión de recursos lingüísticos; en [9] representan los tweets por medio de vectores de palabras ponderados con TF-IDF y son clasificados utilizando algoritmos como máquinas de soporte vectorial (SVM) y regresión logística.

Las aproximaciones híbridas para el análisis de sentimientos también han sido empleadas, en [10] desarrollan una aproximación híbrida para el análisis de sentimiento global en Twitter, mediante el uso de clasificadores y aproximaciones sin supervisión, construidas mediante léxicos de polaridad y estructuras sintácticas.

El uso de lexicones para la tarea de clasificación de polaridad en textos de opiniones o mensajes de redes sociales, tanto en inglés como en español, se ha abordado por [11], quien desarrolló un lexicón con las palabras que co-existen en diversos corpora de opiniones y evalúa su desempeño con el algoritmo de Bayes. Por su parte, en [12] consideran como punto de partida el significado cambiante de una palabra que depende de las relaciones sociales que tiene cada autor, en dicho trabajo se utiliza una red neuronal para la tarea de clasificación. Finalmente, la adaptación de un recurso léxico denominado (ANEW) que originalmente no está destinado al idioma español, pero que [13] han traducido al español, es utilizada para identifican la necesidad de una opinión neutral al reorganizar la escala ANEW, si las palabras del conjunto de prueba no coinciden con el léxico generado, se consideran para la categoría de ninguno (NONE).

\section{Descripción de la arquitectura}

En esta sección se presenta una arquitectura (ver Figura 1) para evaluar el uso del lexicón denominado $M L$-SentiCon en la tarea de clasificación de polaridad a nivel de tweets en español.

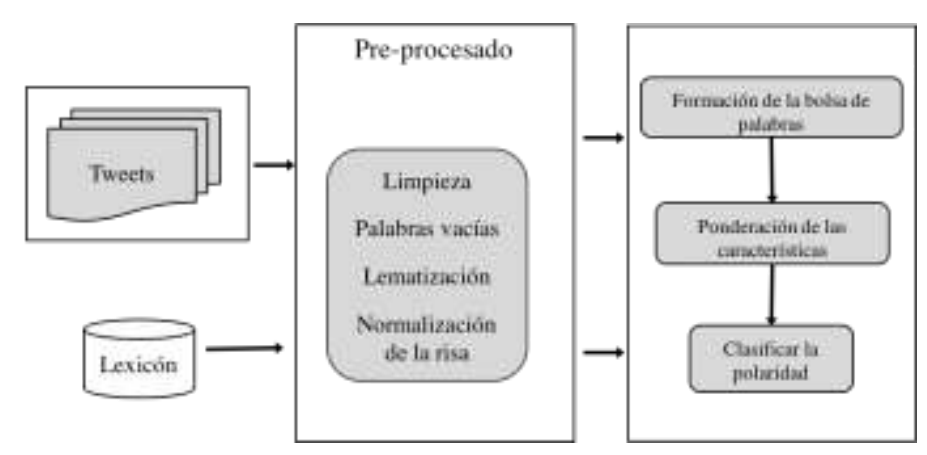

Fig. 1. Arquitectura para la evaluación del lexicón. 


\subsection{Lexicón}

El lexicón evaluado en la arquitectura propuesta es denominado ML-SentiCon [3], el cual está constituido por 11302 entradas o lemas en español. Este recurso contiene lemas polarizados, con valores que van desde -1.0 "negativo" hasta +1.0 "positivo" y, adicionalmente, un valor de desviación estándar que refleja la ambigüedad resultante del cómputo de la polaridad a partir de los valores de los distintos significados posibles del lema. Además, se tiene la categoría gramatical para cada lema, a saber: verbo (v), sustantivo (s), adjetivo (adj), adverbio (adv), determinante (DT).

Este lexicón es generado de manera automática utilizando una versión mejorada del método usado para construir SentiWordNet 3.0 [14].

Las entradas (frases) del lexicón son extraídas, pre-procesada, representadas utilizando el modelo de bolsa de palabras y ponderadas. Ellas son utilizadas por el algoritmo de clasificación para determinar la polaridad del tweet.

\subsection{Pre-procesado de tweets}

Esta tarea de pre-procesado de los textos se aplica a los diversos experimentos realizados, así como, al lexicón utilizado. Por lo tanto, en esta sección se describen las tareas que involucra el pre-procesado de tweets.

La primera tarea es la limpieza de los textos. Para ello se realiza una segmentación por palabras (tokens) y la eliminación de caracteres especiales, como acentos (á, é, í, ó, ú) y signos de puntuación (. , i i ;). Después, las unidades léxicas son filtradas eliminando las ligas (url) a sitios web externos y las menciones de usuarios en Twitter (@). También, se lleva a cabo una normalización de las unidades léxicas resultantes a minúsculas y se eliminan las stopwords, palabras que no aportan significado y por lo tanto, no son funcionales para la identificación de polaridad. Sin embargo, se conservan las palabras de negación (no, ni) o afirmación (si), al ser consideradas como funcionales para la identificación de la polaridad manifestada por un tweet.

Las tareas de normalización de la risa y la lematización de las palabras son aplicadas a los experimentos con los lexicones externos y a los textos de los tweets con la finalidad de encontrar mayor correspondencia al momento de la ponderación de características.

El objetivo de la normalización de la risa es evitar la redundancia en la forma de expresarla. Para ello, se aplican las reglas o patrones mostrados en la Tabla 1, y se sustituyen las diversas formas de expresar risa por el término en común "jaja".

Tabla 1. Normalización de la risa.

\begin{tabular}{ccc}
\hline Patrón & Frase & Risa normalizada \\
\hline$($ ja $)+$ & ja & jaja \\
$(\mathrm{je})+$ & jeje & jaja \\
$(\mathrm{jo})+$ & jojojo & jaja \\
$(\mathrm{ji})+$ & jijijiji & jaja \\
lol & lol & jaja \\
\hline
\end{tabular}


La lematización consiste en obtener la raíz o lema de las palabras, es decir, eliminar los sufijos o flexiones de las palabras. Esto permite agrupar todas las palabras con la misma raíz en una sola representación en el lexicón propio y, mejora el mapeo de los términos de los lexicones externos con los textos de los tweets. Para esta tarea se utiliza la herramienta TreeTagger (Schmid, 1999), la cual tiene soporte para el español.

Tabla 2. Forma enraizada de palabras para el español.

\begin{tabular}{cc}
\hline Palabra & Lema \\
\hline malas & malo \\
sentirán & sentir \\
fueron & ir \\
felices & feliz \\
\hline
\end{tabular}

En la Tabla 2, se ha mostrado un ejemplo de palabras en español tal como aparecen en los textos y con su lema (raíz) que es generada por la herramienta.

\subsection{Extracción de características y ponderación}

La extracción de características se realiza a partir de las entradas del lexicón MLSentiCon pre-procesadas y su pesado es determinado con la métrica TF-IDF considerando los textos de los tweets. Tanto la extracción de características como la ponderación de las mismas se describen detalladamente como sigue.

Para la formación de características, se extraen las entradas del lexicón como unidades. Ellas representan las características para la etapa de clasificación de la polaridad de cada tweet en alguna categoría de acuerdo al conjunto de datos a evaluar. El modelo de bolsa de palabras (bag-of-words) es utilizado para la representación de las entradas de los lexicones.

La ponderación de las características está determinada por la métrica denominada Frecuencia del Término- Frecuencia Inversa en los Documentos (TF-IDF). Dicha métrica se basa en la frecuencia de aparición del término dentro de una colección de textos. Esta ponderación utiliza la frecuencia de aparición de los términos de la bolsa de palabras (lexicón) en un texto, la cual consiste en el número de veces que un término $(t)$ del lexicón aparece en un tweet $(S)$, ver Ecuación 1, y la frecuencia inversa que determina si el término es común en la colección de tweets (Ecuación 2). Esta información se utiliza, entonces, para calcular el valor final de TF-IDF (Ecuación 3):

$$
\begin{gathered}
\operatorname{TF}\left(t_{i}, S_{j}\right)=f\left(t_{i}, S_{j}\right), \\
\operatorname{IDF}\left(t_{i}, S_{j}\right)=\log \frac{|S|}{1+/ s \in S: t_{i} \in_{S} \mid},
\end{gathered}
$$


Fabián Paniagua-Reyes, José A. Reyes-Ortiz, Belem Priego-Sánchez

$$
w_{i j}=T F\left(t_{i}, S_{j}\right) \times \operatorname{IDF}\left(t_{i}, S_{j}\right) .
$$

En esta fase, se obtienen un conjunto de vectores numéricos que representan cada uno de los tweets del conjunto a evaluar, en este caso, las colecciones de tweets en español de la competencia TASS en sus ediciones 2016 y 2017.

\subsection{Clasificación de la polaridad a nivel de tweet}

La tarea clasificación de polaridad a nivel de tweets consiste en determinar la etiqueta (grado de polaridad) de un tweet basándose en su contenido. En esta arquitectura se lleva a cabo la evaluación del lexicón ML-SentiCon con tres conjuntos de datos (tweets en español).

Cada uno de estos conjuntos tienen un número diferente de categorías. Así pues, en su edición 2016 de la competencia del TASS, se proporcionan el mismo conjunto de tweets en dos versiones: una con seis casos de etiquetas: Muy Positivo (P+), Positivo $(\mathrm{P})$, Negativo (N), Muy Negativo (N+) Neutro (NEU) y Ninguna (NONE) y la otra con cuatro casos de etiquetas: Positivo (P), Negativo (N), Neutro (NEU) y Ninguna (NONE). Además, la edición 2017 de TASS proporciona un nuevo conjunto de tweets con cuatro categorías: Positivo (P), Negativo (N), Neutro (NEU) y Ninguna (NONE).

La idea es utilizar un algoritmo de clasificación supervisada para determinar la etiqueta adecuada. Entonces, para esta tarea de clasificación supervisada se utiliza la representación de los tweets en los vectores ponderados con TF-IDF. Para dicha tarea, se utiliza el clasificador denominado Máquinas de Soporte Vectorial y presentado en [15], el cual construye un conjunto de hiperplanos en un espacio n-dimensional con los tweets de entrenamiento, estos hiperplanos son utilizados para predecir la clase de los tweets de prueba.

El clasificador basado en máquinas de soporte vectorial ha demostrado resultados alentadores en la clasificación de textos. La implementación del algoritmo de clasificación se ha llevado a cabo mediante la librería de aprendizaje automático denominada scikit-learn en Python [16].

\section{Configuración experimental}

El objetivo es evaluar el lexicón ML-SentiCon con tres conjuntos de tweets. Para ello, a partir del lexicón, se ha obtenido un conjunto de 3084 lemas o palabras que, después de ser ponderadas, forman una entrada en la matriz de la bolsa de palabras para cada conjunto de datos.

La evaluación del lexicón consiste en comparar los resultados para la tarea de clasificación de polaridad en tres conjuntos de tweets proporcionados por la competencia TASS, dos de la edición 2016 y uno de la edición 2017.

El conjunto de datos del TASS 2016 para seis etiquetas ( $\mathrm{P}+, \mathrm{P}, \mathrm{N}, \mathrm{N}+, \mathrm{NEU}, \mathrm{NONE})$ consta de 7810 tweets de entrenamiento y 2577 tweets de prueba. Mientras que el conjunto de cuatro etiquetas corresponde al subconjunto de tweets con las cuatro etiquetas 
(P, N, NEU, NONE), el cual consta de 6580 tweets de entrenamiento y 2172 tweets de prueba. Finalmente, el conjunto de datos del TASS 2017 consta de 7311 tweets de entrenamiento y 2400 tweets de prueba.

Todos los experimentos fueron llevados a cabo con los parámetros: complejidad o número de hiperplanos a construir: -C 1; parámetro gama (tipo de kernel a utilizar): -K PolyKernel; tamaño de la memoria cache a utilizar: -C 250007; parámetro de tolerancia: -L 0.001; y épsilon: -P 1.0E-12.

\section{Resultados}

Los resultados de la evaluación del lexicón para la clasificación de polaridad se centran en tres conjuntos de tweets: TASS 2016 con seis etiquetas (TASS 2016-6L), TASS 2016 con cuatro etiquetas (TASS 2016-4L) y TASS 2017 con cuatro etiquetas (TASS 2017-4L).

Los resultados de cada conjunto son presentados en términos de Precisión (P), Exhaustividad (R) y la medida F1. Estas métricas comparan los resultados del clasificador a evaluar con los valores externos de confianza (tweets etiquetados), utilizando los siguientes valores: a) Verdadero Positivo (VP) es el número de predicciones correctas del clasificador que corresponden al juicio externo de confianza (tweets etiquetados); Verdadero Negativo (VN) es el número de predicciones correctas del clasificador de polaridades que no corresponden al juicio externo de confianza; Falso Positivo (FP) corresponde al número predicciones incorrectas del clasificador que corresponden al juicio externo de confianza; y, finalmente Falso Negativo (FN) es el número de predicciones incorrectas del clasificador que no corresponden al juicio externo de confianza.

Bajo estos criterios, se emplea la Precisión (P) para evaluar los algoritmos en términos de los valores de predicciones positivas, la cual se define, en la Ecuación 4, como:

$$
P=\frac{V P}{V P+F P} .
$$

También, se utiliza el Exhaustividad (R) para expresar la tasa de correspondencias correctas con las opiniones de textos preclasificados de manera externa con una alta confianza (Ecuación 5):

$$
R=\frac{V P}{V P+F N}
$$

Finalmente, la medida F (F1) que representa la media armónica entre Precisión y Cobertura, la cual tiene como fundamento el obtener un valor único ponderado entre ellas (Ecuación 6):

$$
F 1=2 * \frac{P * R}{P+R} .
$$

Los resultados de evaluar el lexicón ML-SentiCon para el conjunto TASS 2016 con seis etiquetas (TASS 2016-6L), en la tarea de clasificación de polaridad, se muestra en la Tabla 3. 
Fabián Paniagua-Reyes, José A. Reyes-Ortiz, Belem Priego-Sánchez

Tabla 3. Resultados para el conjunto TASS 2016-6L.

\begin{tabular}{cccc}
\hline Etiqueta & $\mathrm{P}$ & $\mathrm{R}$ & $\mathrm{F} 1$ \\
\hline $\mathrm{P}+$ & 0.508 & 0.506 & 0.507 \\
$\mathrm{P}$ & 0.488 & 0.489 & 0.488 \\
$\mathrm{~N}$ & 0.433 & 0.430 & 0.431 \\
$\mathrm{~N}+$ & 0.413 & 0.411 & 0.412 \\
$\mathrm{NEU}$ & 0.375 & 0.373 & 0.374 \\
$\mathrm{NONE}$ & 0.103 & 0.104 & 0.103 \\
\hline
\end{tabular}

Los resultados de evaluar el lexicón para el conjunto TASS 2016 con cuatro etiquetas (TASS 2016-4L), se muestra en la Tabla 4.

Tabla 4. Resultados para el conjunto TASS 2016-4L.

\begin{tabular}{cccc}
\hline Etiqueta & $\mathrm{P}$ & $\mathrm{R}$ & $\mathrm{F} 1$ \\
\hline $\mathrm{P}$ & 0.540 & 0.540 & 0.540 \\
$\mathrm{~N}$ & 0.529 & 0.528 & 0.529 \\
$\mathrm{NEU}$ & 0.497 & 0.495 & 0.496 \\
NONE & 0.165 & 0.163 & 0.164 \\
\hline
\end{tabular}

Los resultados de evaluar el lexicón para el conjunto TASS 2017 con cuatro etiquetas (TASS 2017-4L), se muestra en la Tabla 5.

Tabla 5. Resultados para el conjunto TASS 2017-4L.

\begin{tabular}{cccc}
\hline Etiqueta & $\mathrm{P}$ & $\mathrm{R}$ & $\mathrm{F} 1$ \\
\hline $\mathrm{P}$ & 0.553 & 0.551 & 0.552 \\
$\mathrm{~N}$ & 0.539 & 0.536 & 0.537 \\
$\mathrm{NEU}$ & 0.509 & 0.509 & 0.509 \\
$\mathrm{NONE}$ & 0.168 & 0.166 & 0.167 \\
\hline
\end{tabular}

La categoría de tweets con la etiqueta NONE tuvo un pobre desempeño en los tres conjuntos de datos, considerando el lexicón ML-SentiCon. Esto se debe, en gran medida, a que dicho lexicón carece de palabras para esta categoría, es decir, sin carga de polaridad o sin valor. Todas las entradas en el lexicón tienen un valor entre -1 y +1 .

Finalmente, la Tabla 6 muestra la comparativa que permite evaluar el lexicón en los tres subconjuntos: TASS 2016 con seis etiquetas (TASS 2016-6L), TASS 2016 con cuatro etiquetas (TASS 2016-4L) y TASS 2017 con cuatro etiquetas (TASS 2017-4L). Estos resultados son mostrados utilizando las versiones macro (promedios) de Precisión (Macro-P), Exhaustividad (Macro-R) y medida F1 (Macro-F1) con la finalidad de proporcionar una comparación comprensiva de los resultados. 
Evaluando un lexicón para la clasificación de polaridad a nivel de Tweet en español

Tabla 6. Resultados del lexicón en tres conjuntos.

\begin{tabular}{cccc}
\hline Conjunto de datos & Macro-P & Macro-R & Macro-F1 \\
\hline TASS 2016-6L & 0.387 & 0.375 & 0.386 \\
TASS 2016-4L & 0.433 & 0.431 & 0.432 \\
TASS 2017-4L & 0.442 & 0.441 & 0.441 \\
\hline
\end{tabular}

Los resultados mostrados en la Tabla 6 hacen constar que el lexicón ML-SentiCon tiene un mejor desempeño, en cuanto a la tarea de clasificación de polaridad, en el conjunto de la competencia TASS en su edición 2017 considerando cuatro etiquetas para los tweets: Positivo (P), Negativo (N), Neutro (NEU) y Ninguna (NONE).

\section{Conclusiones}

Este artículo ha presentado una arquitectura para evaluar el uso del lexicón denominado ML-SentiCon en la tarea de clasificación de polaridad en mensajes de tweets en español. La arquitectura presentada considera como componente al lexicón y lo evalúa en tres conjuntos de tweets: TASS 2016 con seis etiquetas, TASS 2016 con cuatro etiquetas y TASS 2017 con cuatro etiquetas. La clasificación de la polaridad se lleva a cabo con el algoritmo Máquinas de Soporte Vectorial utilizando un modelo de bolsa de palabras y una ponderación TF-IDF.

La evaluación del lexicón ha hecho evidente que, en los tres conjuntos de datos, la categoría de NONE presente los peores resultados en cuanto a medida F1. Esto se debe, en gran medida, a que el lexicón no contiene entradas para dicha categoría. Es decir, todas las palabras tienen un valor de polaridad asignado.

Con las pruebas de evaluación del lexicón, también, ha resultado que el lexicón MLSentiCon tiene un mejor desempeño, en cuanto a la tarea de clasificación de polaridad, en el conjunto de la competencia TASS en su edición 2017 que con el resto de los conjuntos de tweets.

Este trabajo ha contribuido en contar con un recurso de análisis comparativo de recursos lingüísticos para el español en la tarea de análisis de sentimientos.

Una línea de investigación como trabajo a futuro, se puede centrar en la categoría de NONE y proponer un enfoque para considerar entradas en los lexicones sin polaridad. Como trabajo futuro, también, se puede experimentar con más lexicones con entradas polarizadas o categorizadas (Positivas Negativas, Neutras) para el español y extender la evaluación hacia otros conjuntos de tweets. Finalmente, considerar la clasificación de tweets por tema o tópico considerando el lexicón ML-SentiCon, puede resultar de gran utilidad.

Agradecimientos. Este trabajo fue apoyado, parcialmente, por la Red Temática en Tecnologías del Lenguaje de CONACyT. Los autores agradecen a la Universidad Autónoma Metropolitana unidad Azcapotzalco por el apoyo recibido para la realización de este trabajo. 


\section{Referencias}

1. Martínez-Cámara, E., Díaz-Galiano, M. C., García-Cumbreras, M. Á., García-Vega, M., Villena-Román, J: Overview of TASS 2017. In: Proceedings of TASS 2017: Workshop on Semantic Analysis at SEPLN (TASS 2017), CEUR Workshop Proceedings, España, 13-21 (2017)

2. García-Cumbreras, M. Á., Villena-Román, J., Cámara, E. M., Díaz-Galiano, M. C., MartínValdivia, M. T., López, L. A. U.: Overview of TASS 2016. In: Proceedings of TASS 2016: Workshop on Sentiment Analysis at SEPLN colocated with 31st SEPLN Conference. CEUR Workshop Proceedings, España, 13-21 (2016)

3. Cruz, F. L., Troyano, J. A., Pontes, B., Ortega, F. J.: ML-SentiCon: Un lexicón multilingüe de polaridades semánticas a nivel de lemas. Procesamiento del Lenguaje Natural, 53, 113$120(2014)$

4. Vilares, D., Doval, Y., Alonso, M. A., Gómez-Rodríguez, C.: LyS at TASS 2015: Deep Learning Experiments for Sentiment Analysis on Spanish Tweets. In: Proceedings of TASS 2015: Workshop on Sentiment Analysis at SEPLN co-located with 31st SEPLN Conference (SEPLN 2015), CEUR Workshop Proceedings, España, 47-52 (2015)

5. Díaz-Galiano, M. C., Montejo-Ráez, A.: Participación de SINAI DW2Vec en TASS 2015. In: Proceedings of TASS 2015: Workshop on Sentiment Analysis at SEPLN co-located with 31st SEPLN Conference (SEPLN 2015), CEUR Workshop Proceedings, España, 59-64 (2015)

6. Montejo-Ráez, A., Díaz-Galiano, M. C.: Participación de SINAI en TASS 2016. In: Proceedings of TASS 2016: Workshop on Sentiment Analysis at SEPLN colocated with 31st SEPLN Conference, CEUR Workshop Proceedings, España, 41-45 (2016)

7. Murillo, E. C., Raventós, G. M.: Evaluación de Modelos de Representación del Texto con Vectores de Dimensión Reducida para Análisis de Sentimiento. In: Proceedings of TASS 2016: Workshop on Sentiment Analysis at SEPLN co-located with 31st SEPLN Conference, CEUR Workshop Proceedings, España, 23-28 (2016)

8. Martínez-Cámara, E., García Cumbreras, M. Á., Martín-Valdivia, M. T., Ureña-López, L. A.: SINAI-EMMA: Vectores de Palabras para el Análisis de Opiniones en Twitter. In: Proceedings of TASS 2015: Workshop on Sentiment Analysis at SEPLN co-located with 31st SEPLN Conference (SEPLN 2015), CEUR Workshop Proceedings, España, 41-46 (2015)

9. Quirós, A., Segura-Bedmar, I., Martínez, P.: LABDA at the 2016 TASS Challenge Task: Using Word Embeddings for the Sentiment Analysis Task. In: Proceedings of TASS 2016: Workshop on Sentiment Analysis at SEPLN co-located with 31st SEPLN Conference, CEUR Workshop Proceedings, Españ, 29-33a (2016)

10. Alvarez-López, T., Juncal-Martínez, J., Gavilanes, M. F., Costa-Montenegro, E., GonzálezCastano, F. J., Cerezo-Costas, H., Celix-Salgado, D.: GTI-Gradiant at TASS 2015: A Hybrid Approach for Sentiment Analysis in Twitter. In: Proceedings of TASS 2015: Workshop on Sentiment Analysis at SEPLN co-located with 31st SEPLN Conference (SEPLN 2015), CEUR Workshop Proceedings, España, 35-40 (2015)

11. Eisenstein, J.: Unsupervised Learning for Lexicon-Based Classification. In: Proceedings of the Thirty-First AAAI Conference on Artificial Intelligence (AAAI-17), AAAI Publications, USA, 3188-3194 (2017)

12. Yang, Y., Eisenstein, J.: Overcoming Language Variation in Sentiment Analysis with Social Attention. Transactions of the Association for Computational Linguistics 5, 259-307 (2017) 
13. Henriquez, M., Guzman, J. A., Salcedo, D.: Minería de Opiniones basado en la adaptación al español de ANEW sobre opiniones acerca de hoteles. Procesamiento del Lenguaje Natural, 56, 25-32 (2016)

14. Baccianella, S., Esuli, A., Sebastiani, F.: Sentiwordnet 3.0: An enhanced lexical resource for sentiment analysis and opinion mining. In: Proceedings of the Seventh conference on International Language Resources and Evaluation (ELRA), Malta, 2200-2204 (2010)

15. Chang, Ch., Lin, Ch.: LIBSVM - A Library for Support Vector Machines. ACM Transactions on Intelligent Systems and Technology 2(3), 27-28 (2001)

16. Pedregosa, F., Varoquaux, G., Gramfort, A., Michel, V., Thirion, B., Grisel, O., Blondel, M. Prettenhofer, P., Weiss, R., Dubourg, V., Vanderplas, J., Passos, A., Cournapeau, D., Brucher, M., Perrot, M., Duchesnay, E.: Scikit-learn: Machine Learning in Python. Journal of Machine Learning Research 12, 2825-2830 (2011) 